\title{
Natural Frequency of Beams with Embedded Piezoelectric Sensors and Actuators
}

\author{
C. Della \& D. Shu \\ School of Mechanical and Aerospace Engineering, Nanyang Technological University, Singapore
}

ABSTRACT: A mathematical model is developed to study the natural frequency of beams with embedded piezoelectric sensors and actuators. The piezoelectric sensors/actuators in a non-piezoelectric matrix (host beam) are analyzed as two inhomogeneity problems by using Eshelby's equivalent inclusion method. The natural frequency of the beam is determined from the variational principle in Rayleigh quotient form, which is expressed as functions of the elastic strain energy and dielectric energy of the piezoelectric sensors/actuators. The Euler-Bernoulli beam theory and Rayleigh-Ritz approximation technique are used in the present analysis. Parametric studies show that the size, volume fraction and location of the piezoelectric inclusions significantly influence the natural frequency of the beam.

Keywords: Natural frequency; Beam; Piezoelectric sensors and actuators; Eshelby's equivalent inclusion method.

\section{INTRODUCTION}

Smart structures are important because of their relevance to structural health monitoring, structural vibration control and transportation engineering. This is because these systems incorporate particular functions such as sensing, processing and actuation. The use of piezoelectric materials is a primary focus in the study of smart materials since these materials can function both as sensors and actuators (Tani et al., 1998).

Vibration control and active damping are among the most studied areas using smart materials and structures (Chee, 1998). Extensive research has been done on the vibration control and suppression of structures using piezoelectric materials, as evident from numerous review articles (see for example Ahmadian and DeGuillio, 2001; Irschik, 2002; Sunar and Rao, 1999; Wetherhold and Aldrahem, 2001). Many mathematical models for laminates and structures with piezoelectric sensors and/or actuators have been presented in the literature, and reviews of these models have been presented (see for example Chee et al.,1998; Chopra, 2002).

An analysis of these reviews indicates that piezoceramic materials are widely used as sensors and/or actuators. They are either in the form of patches or lamina. The piezoelectric patches are either bonded to or embedded within the structures, whereas the piezoelectric lamina are stacked together with a substrate laminae to form a piezoelectric composite laminate. However, there are several factors that limit the use of piezoceramic materials, such as their brittle nature and low tensile strength, therefore limiting their ability to conform to curved shapes, and the large add-on mass associated with using typical lead-based piezoceramic. The use of arrays of piezoelectric sensors and actuators embedded within the structure would remedy the above mentioned restric- tions. Due to their small size, these sensors/actuators have the flexibility to conform to curved shapes, and they add little weight to the structure. In addition, these piezoelectric sensors and actuators can be tailored to achieve a particular smart structure design.

Because of the small size of the piezoelectric sensors and actuators relative to the size of the host structure, these sensors and actuators can be analyzed as inclusions in a non-piezoelectric matrix (host structure) by using a micromechanics approach. Fan and Qin (1995) analyzed a piezoelectric sensor embedded in a non-piezoelectric elastic matrix by using Eshelby's equivalent inclusion method (Eshelby, 1957; Mura, 1987). The piezoelectric problem was decoupled into an elastic inclusion problem and a dielectric inclusion problem connected by some eigenstrain and eigen-electricfield.

Irschik et al. (1998) analyzed the piezoelectric actuation for vibration and shape control of structures as an eigenstrain actuation. An eigenstrain technique was presented by Alghamdi and Dasgupta (1993a, $1993 \mathrm{~b}$ ) for the vibration of beams with embedded arrays of piezoelectric sensors and actuators. The embedded sensors and actuators were analyzed as piezoelectric ellipsoidal inclusion in an infinite matrix (host beam) by using Eshelby's equivalent inclusion method. Using the variational principle in Rayleigh quotient form, they formulated an equation for the natural frequency of the beam, which was expressed as functions of the elastic strain energy and dielectric energy of the beam. However, the piezoelectric inclusions were analyzed as elastic inclusions only, thereby neglecting the dielectric effects of the piezoelectric inclusions. The influence of the mechanicalelectrical coupling of the piezoelectric sensors on the natural frequency was also neglected in their analyses.

In this research, a mathematical model for the vibration of beams with embedded arrays of piezoelec- 
tric sensors and actuators is presented. The piezoelectric sensors and actuators are analyzed as inhomogeneous ellipsoidal inclusions in a nonpiezoelectric matrix (host beam) by using Eshelby's equivalent inclusion method (Eshelby, 1957; Mura, 1987). The formulation for the piezoelectric inclusion problem is decoupled into two equivalent inclusion problems, an elastic problem and a dielectric problem. An equation for the natural frequency of the beam is determined using the variational principle in Rayleigh quotient form, which is expressed as functions of the elastic strain energy and dielectric energy of the piezoelectric inclusions. These energies are derived using Mura's formulation for inhomogeneous inclusions. The Euler-Bernoulli beam theory and Rayleigh-Ritz approximation technique are used in the present analysis. In addition, the influences of the size, volume fraction and location of the piezoelectric inclusions on the natural frequency of the beam are studied.

The research is presented as follows. First, the mathematical modeling is presented, which begins with the formulation of the equation for the natural frequency of a piezoelectric body. This is followed the analysis of a non-piezoelectric matrix with piezoelectric ellipsoidal inclusions using Eshelby's equivalent inclusion method. The energies of the piezoelectric inclusions are then formulated, and an explicit solution for the natural frequency of a beam with piezoelectric inclusions is obtained. Next, using the mathematical model presented, In addition, the influences of the size, volume fraction and location of the piezoelectric inclusions on the natural frequency of the beam are studied.

\section{MATHEMATICAL MODELING}

\subsection{Variational principle in Rayleigh quotient form}

Let $\Omega$ be a region occupied by a piezoelectric body and $S$ be boundary surface of $\Omega$. The constitutive relations for a linear piezoelectric material are

$$
\begin{array}{ll}
\sigma_{i j}=C_{i j m n} \varepsilon_{m n}-e_{n i j} E_{n} & \text { in } \Omega \\
D_{i}=e_{i m n} \varepsilon_{m n}+\kappa_{i n} E_{n} & \text { in } \Omega
\end{array}
$$

where $\sigma_{i j}, \varepsilon_{i j}, E_{i}$ and $D_{i}$ are the stress tensor, strain tensor, electric field vector and the electric displacement vector, respectively. $C_{i j m n}, e_{n i j}$ and $\kappa_{i n}$ are the elastic stiffness tensor, the piezoelectric tensor and permittivity tensor, respectively.

For the time-harmonic free vibration of a piezoelectric body with circular frequency $\omega$, the governing equations and boundary conditions in rectangular Cartesian coordinates are (Tiersten, 1969)

$$
\begin{gathered}
\sigma_{i j, j}=\left(C_{i j m n} u_{m, n}+e_{n i j} \phi_{, n}\right)_{, j}=\rho \omega^{2} u_{i} \text { in } \Omega \\
D_{i, i}=\left(e_{i m n} u_{m, n}-\kappa_{i n} \phi_{, n}\right)_{, i}=0 \text { in } \Omega
\end{gathered}
$$

$u_{i}=0$ on $\mathrm{S}$

$\sigma_{i j} n_{j}=\left(C_{i j m n} u_{m, n}+e_{n i j} \phi_{, n}\right) n_{j}=0$ on $\mathrm{S}$

$\phi=0$ on $\mathrm{S}$

$D_{i} n_{i}=\left(e_{i m n} u_{m, n}-\kappa_{i n} \phi_{, n}\right) n_{i}=0 \quad$ on $\mathrm{S}$

where $n_{j}$ is the outward pointing unit normal vector. Conventional indicial notation is utilized where repeated subscripts are summed over the range 1-3 and the comma denotes partial differentiation.

For stationary solutions, the Rayleigh quotient can be expressed as

$$
\omega^{2}=\frac{\frac{1}{2} \int_{V} C_{i j m n} \varepsilon_{i j} \varepsilon_{m n} d V+\frac{1}{2} \int_{V} \kappa_{i n} E_{i} E_{n} d V}{\frac{1}{2} \int_{V} \rho u_{i} u_{i} d V}
$$

which was obtained by Eernisse (1967) and Yang and Batra (1993). The numerator in Equation 9 is the internal energy of the system, which is the sum of the elastic strain energy and the dielectric energy. These energies will be analyzed using a micromechanics approach.

\subsection{Energies of piezoelectric inclusion}

The elastic strain energy of the piezoelectric inclusion is obtained using the method presented in Mura (1987) for inhomogeneous inclusions. When a body $D$, containing an inhomogeneous inclusion $\Omega$ with eigenstrain $\varepsilon_{i j}^{p}$, is subjected to an external force $F_{i}$, the elastic strain energy is

$$
W_{e}=\frac{1}{2} \int_{D}\left(\sigma_{i j}^{0}+\sigma_{i j}\right)\left(u_{i, j}^{0}+u_{i, j}-\varepsilon_{i j}^{p}\right) d D
$$

where $\sigma_{i j}^{0}$ is the uniform far field loading, $\varepsilon_{m n}^{0}$ is the strain corresponding to the far field loading, $\sigma_{i j}$ and $\varepsilon_{m n}$ are the disturbance stress and strains due to the presence of inhomogeneity, respectively, and $\varepsilon_{m n}^{p}$ is the eigenstrain due to the electromechanical coupling of the piezoelectric actuator or the eigenstrain actuation (Irschik, et al., 1998). Applying Mura's (1987) energy of inhomogeneous inclusion, Equation 10 can be expressed as

$$
W_{e}=\frac{1}{2} \int_{D} \sigma_{i j}^{0} \varepsilon_{i j}^{0} d D+\frac{1}{2} \int_{\Omega} \sigma_{i j}^{0} \varepsilon_{i j}^{*} d D-\frac{1}{2} \int_{\Omega} \sigma_{i j} \varepsilon_{i j}^{p} d D
$$

where the fictitious eigenstrain $\varepsilon_{i j}^{*}$ can be determined from

$$
C_{i j m n}^{*}\left(\varepsilon_{m n}^{0}+S_{m n a b} \varepsilon_{a b}^{*}\right)=C_{i j m n}\left(\varepsilon_{m n}^{0}+S_{m n a b} \varepsilon_{a b}^{*}-\varepsilon_{m n}^{*}\right)
$$


and $\sigma_{i j}$ can be determined from

$$
\sigma_{i j}=C_{i j m n}^{*}\left(S_{m n a b} \varepsilon_{a b}^{* *}-\varepsilon_{a b}^{p}\right)=C_{i j m n}\left(S_{m n a b} \varepsilon_{a b}^{* *}-\varepsilon_{a b}^{* *}\right)
$$

The elastic Eshelby tensor $S_{m n a b}$ is only a function of the matrix Poisson's ratio and the ellipsoidal aspect ratios. The components of the Eshelby tensor can be found in Mura (1987).

Similarly, the dielectric energy can be determined using Mura's method for elastic inhomogeneous inclusions. The dielectric energy is given by

$W_{d}=\frac{1}{2} \int_{\Omega}\left(D_{i}^{0}+D_{i}\right)\left(E_{i}^{0}+E_{i}-E_{i}^{p}\right) d D$

which can be expressed as

$W_{d}=\frac{1}{2} \int_{\Omega} D_{i}^{0} E_{i}^{0} d D-\frac{1}{2} \int_{\Omega} D_{i} E_{i}^{p} d D$

where $D_{i}^{0}$ and $E_{n}^{0}$ are the known far fields, and $E_{n}^{p}$ is caused by the mechanical-electrical coupling and where

$D_{i}^{0}=-\kappa_{i n}^{*} \phi_{, n}^{0}=\kappa_{i n}^{*} E_{n}^{0}$

and

$$
D_{i}^{0}+D_{i}=\kappa_{i n}^{*}\left(E_{n}^{0}+E_{n}-E_{n}^{p}\right)
$$

where $D_{i}$ can be determined from

$$
D_{i}=\kappa_{i n}^{*}\left(s_{n a} E_{a}^{* *}-E_{i}^{p}\right)
$$

The electric Eshelby tensor, $s_{n b}$, is only a function of the ellipsoidal aspect ratio and the components are presented in Hatta and Taya (1986). The details of the formulation can be found in Fan and Qin (1995).

\subsection{Natural frequency of a beam with piezoelectric inclusions}

Figure 1 shows a beam with piezoelectric inclusions. The piezoelectric inclusions in the one of the rows are considered to act as sensors and the other row, as actuators. Bending the beam induces strain in the sensors and it produces an output, which is used in a closed-loop constant-feedback-gain control circuit to activate the corresponding actuators. The actuation induces strain along the length of the beam causing an effect opposite to that caused by the initial bending. This results in a stiffening of the beam and an accompanying increase in the natural frequency (Alghamdi and Dasgupta, 1993a, 1993b).

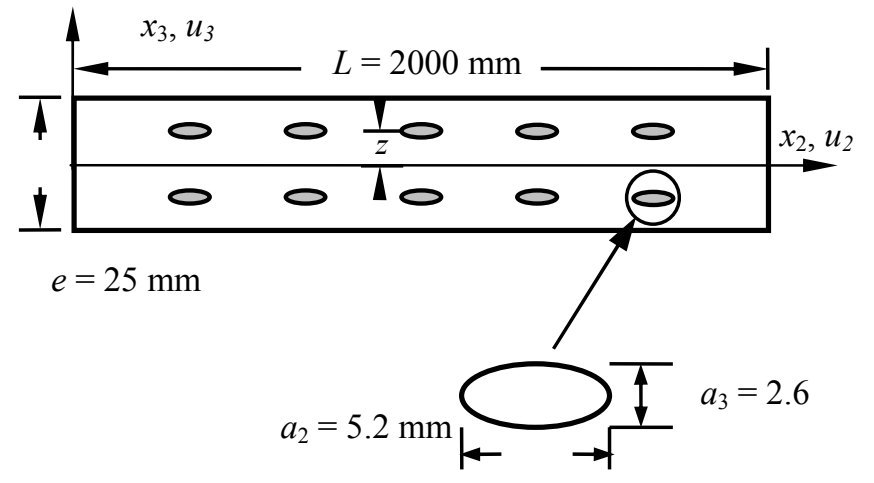

Figure 1. Dimensions of the beam and piezoelectric materials.

The Rayleigh quotient in Equation 9 is used here to determine the natural frequency of a nonpiezoelectric beam with piezoelectric inclusions, which can be written as

$$
\omega^{2}=\frac{\frac{1}{2} \int_{D} C_{i j m n} \varepsilon_{i j}^{0} \varepsilon_{m n}^{0} d D+\frac{1}{2} \int_{\Omega} C_{i j m n} \varepsilon_{i j}^{*} \varepsilon_{m n}^{0} d D}{\frac{1}{2} \int_{V} \rho u_{i} u_{i} d V}
$$

$$
-\frac{\frac{1}{2} \int_{\Omega} C_{i j m n}^{*} \varepsilon_{i j}^{p}\left(S_{m n a b} \varepsilon_{a b}^{* *}-\varepsilon_{m n}^{p}\right) d D}{\frac{1}{2} \int_{V} \rho u_{i} u_{i} d V}
$$

$$
+\frac{\frac{1}{2} \int_{\Omega} \kappa_{i n}^{*} E_{i}^{0} E_{n}^{0} d D-\frac{1}{2} \int_{\Omega} \kappa_{i n}^{*} E_{i}^{p}\left(s_{n a} E_{a}^{* *}-E_{n}^{p}\right) d D}{\frac{1}{2} \int_{V} \rho u_{i} u_{i} d V}
$$

In order to perform the integration in Equation 18, we make the following assumptions:

a. The Euler-Bernoulli beam theory and plane stress assumption are used.

b. Each of the embedded piezoelectric sensors and actuators is assumed to be a piezoelectric cylinder with elliptical cross-section, whose polarization is oriented along the thickness of the beam ( $x_{3}$-axis).

c. The actuator will have both eigenstrain actuation and fictitious eigenstrain, whereas the sensor will only have fictitious eigenstrain.

d. Both sensors and actuators will have real eigen-electricfield due to the direct piezoelectric effect (applied stress on piezoelectric materials induces electric polarization). However, for the sensors, the fictitious eigenelectricfield is zero, since the electric field is applied to the piezoelectric actuator. 
Using the Rayleigh-Ritz technique, for a simplysupported beam, the transverse displacement function is given by

$u_{3}=\sum_{n=1}^{\infty} a_{n} \sin \left(\omega_{n} t\right) \sin \left(\frac{n \pi x_{2}}{L}\right)$

where the $x_{2}$-axis is oriented along the length of the beam, $u_{3}$ is the transverse deflection in the $x_{3}$ direction, $\omega_{n}$ and $a_{n}$ are the natural frequency and amplitude of the nth mode. Only the fundamental frequency $(n=1)$ is of interest in this study. In view of the Euler-Bernoulli beam theory and plane stress assumptions, the only non-zero term in the bending strain field $\varepsilon_{i j}^{0}$ is $\varepsilon_{2}^{0}$ and is determined by

$\varepsilon_{2}^{0}=-z \frac{\partial^{2} u_{3}}{\partial x_{2}{ }^{2}}=z \frac{\pi^{2}}{L^{2}} a_{1} \sin \left(\omega_{1} t\right) \sin \left(\frac{\pi x_{2}}{L}\right)$

where $z$ is the distance of the piezoelectric device from the neutral axis of the beam.

The only non-zero component of the actuation voltage vector is $E_{3}^{0}$ and is proportional to the bending strain $\varepsilon_{2}^{0}$. The eigenstrain actuation $\varepsilon_{i j}^{p}$ is determined by

$\varepsilon_{m n}^{p}=d_{k m n} E_{k}^{0}$

The non-zero terms of the eigenstrain actuation $\varepsilon_{m n}^{p}$ expressed in contracted Voight notation is

$\varepsilon_{q}^{p}=d_{3 q} E_{3}^{0} \quad(q=1-6)$

The eigen-electricfield due to the mechanicalelectrical coupling $E_{k}^{p}$ is determined by

$E_{k}^{p}=-\left[\kappa_{i k}^{*}\right]^{-1} e_{i m n}\left(\varepsilon_{m n}+\varepsilon_{m n}^{0}\right)$

and the non-zero terms are

$$
E_{3}^{p}=-\frac{e_{3 q}}{\kappa_{33}^{*}}\left(\varepsilon_{q}+\varepsilon_{q}^{0}\right) \quad(q=1-6)
$$

\section{RESULTS AND DISCUSSIONS}

This section presents the results obtained using the mathematical model described above to study a simply supported beam with embedded piezoelectric sensors and actuators. The host beam is made of Alplex material and piezoelectric sensors/actuators are made of PZT-5H. Table 1 presents the properties of Alplex and PZT-5H, which is poled along the $x_{3}$ axis. The dimensions of the beam and the piezoelectric inclusions are shown in Fig. 1.

The normalized fundamental frequency $\omega / \omega_{0}$ as a function of the actuator location is shown in Fig. 2, where $\omega_{0}$ is the fundamental frequency of the beam without the actuation effect. The number of actuators, $n$, is identical to the number of sensors. It can be seen that the frequency $\omega / \omega_{0}$ increases as the actuator is located nearer to the beam surface. The frequency further increases with increasing piezoelectric volume fraction $v_{f}$ and $\varepsilon_{2}^{p} / \varepsilon_{2}^{0}$, where $\varepsilon_{2}^{p} / \varepsilon_{2}^{0}$ is the normalized eigenstrain actuation.

Table 1. Properties of the the piezoelectric material poled along $x_{3}$-axis.

\begin{tabular}{lccccccc}
\hline & $\begin{array}{c}\mathrm{E} \\
(\mathrm{GPa})\end{array}$ & & $d_{31}$ & $\begin{array}{c}d_{33} \\
\left(10^{-12} \mathrm{~m} / \mathrm{V}\right)\end{array}$ & $\begin{array}{c}\kappa_{11} \\
\left(10^{-10} \mathrm{C} / \mathrm{Vm}\right)\end{array}$ \\
\hline$\overline{\text { PZT-5H }}$ & 64 & 0.39 & -274 & 593 & 741 & 150 & 66 \\
ALPLEX & 3.5 & 0.35 & - & - & - & - & - \\
\hline
\end{tabular}

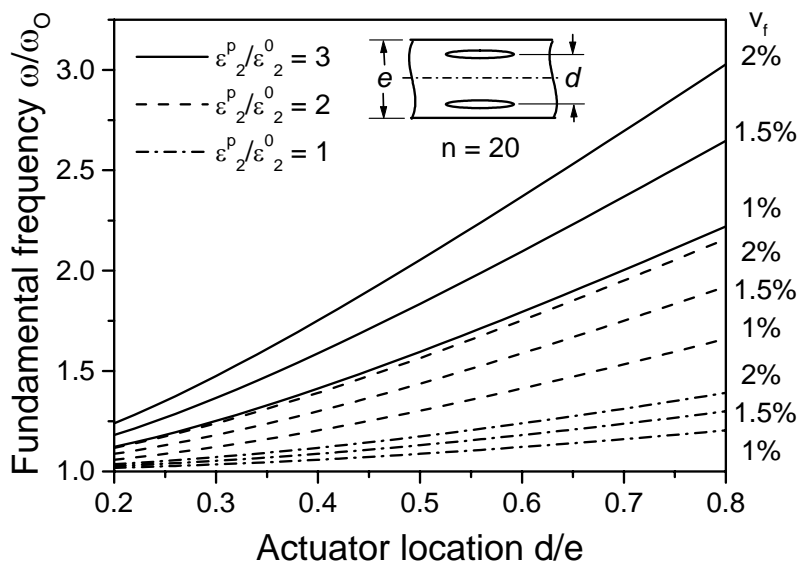

Figure 2. Influence of the actuator location, $d / e$, on the fundamental frequency of the beam, $\omega / \omega_{0}$, with various piezoelectric volume fraction $v_{f}$.

Figure 3 shows the influence of the aspect ratio of the ellipsoidal piezoelectric device $a_{2} / a_{3}$ on the fundamental frequency of the beam for different piezoelectric volume fraction $v_{f}$. It can be seen that the use of a flat piezoelectric device increases the natural frequency. Furthermore, the influence of $a_{2} / a_{3}$ becomes more obvious at higher $\varepsilon_{2}^{p} / \varepsilon_{2}^{0}$. However, the influence of the aspect ratio $a_{2} / a_{3}$ on the frequency is less significant for $\varepsilon_{2}^{p} / \varepsilon_{2}^{0}=1$.

\section{CONCLUSIONS}

A mathematical model has been developed to study the natural frequency of beams with embedded piezoelectric sensors and actuators. The variational principle in Rayleigh quotient form and the Eshelby's equivalent inclusion method have been utilized to obtain an explicit solution for the natural frequency of a beam with embedded piezoelectric sensors and actuators. This equation was used to study the influence of the piezoelectric actuator on the natural frequency of the beam. The present solu- 
tion is general and can be extended to include other inclusions, such as cracks with small volume fraction.

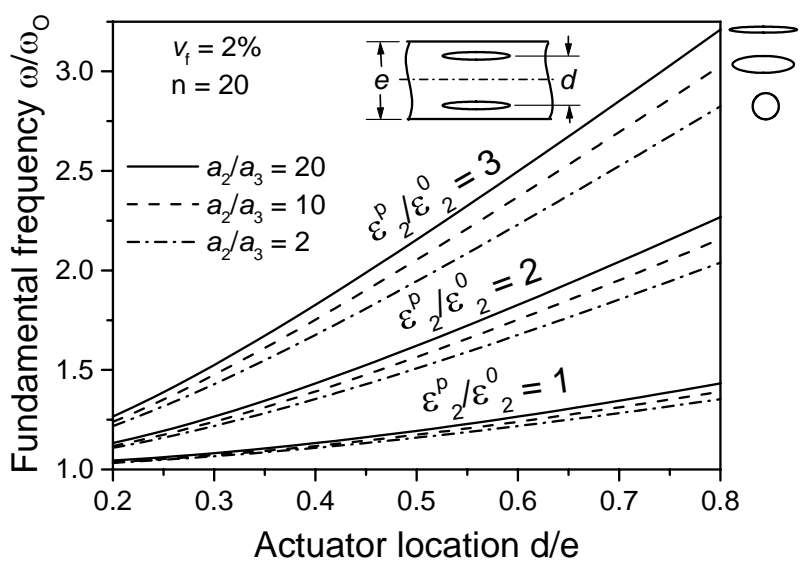

Figure 3. Influence of the actuator location, $d / e$, on the fundamental frequency of the beam, $\omega / \omega_{0}$, with various aspect ratio $a_{2} / a_{3}$ of the piezoelectric sensors and actuators.

\section{REFERENCES}

Ahmadian, M. and DeGuilio, A.P., 2001. Recent advances in the use of piezoceramics for vibration suppression. Shock and Vibration Digest 33(1): 15-22.

Alghamdi, A. and Dasgupta, A., 1993a. Interaction mechanics between embedded microactuators and the surrounding host in adaptive structures. In Nesbitt W. Hagood (Ed), Proc. SPIE Vol. 1917, Smart Struct. Mater. 1993: Smart Struct. Intell. Sys., 317-328.

Alghamdi, A. and Dasgupta, A., 1993b. Micromechanical dynamic analysis of an adaptive beam with embedded distributions of piezoelectric actuator/sensor devices. In Proc. ASME Winter Annual Meeting, New Orleans, LA, November, 121-128.

Chee, C.Y.K., Tong, L.T. and Steven, G.P., 1998. A review on the modeling of piezoelectric sensors and actuators incorporated in intelligent structures. Journal of Intelligent Material Systems and Structures 9(1): 3-19.

Chopra, I., 2002. Review of state of art of smart structures and integrated systems. AIAA Journal 40(11): 2145-2187.

Eernisse, E.P., 1967. Variational method for electroelastic vibration analysis. IEEE Transactions of Sonics and Ultrasonics 14(4): 153-160.

Eshelby, J.D., 1957. The determination of the elastic field of the ellipsoidal inclusion and related problems. Proceedings of the Royal Society of London A 241(1226): 376-396.

Fan, H. and Qin, S., 1995. A piezoelectric sensor embedded in a non-piezoelectric matrix. International Journal of Enginering Sciences 33(3): 379-388.

Irschik, H., 2002. A review on static and dynamic shape control of structures by piezoelectric actuation. Engineering Structures 24(1): 5-11.

Irschik, H., Krommer, M., Belyaev, A.K. and Schlacher, K., 1998. Shaping of piezoelectric sensors/actuators for vibrations of slender beams: coupled theory and inappropriate shape functions. Journal of Intelligent Material Systems and Structures 9(7): 546-554.

Mura, T., 1987. Micromechanics of defects in solids 2nd ed. Martinus Nijoff.

Sunar, M. and Rao, S.S., 1999. Recent advances in sensing and control of flexible structures via piezoelectric materials technology. Applied Mechanics Reviews 52(1): 1-16.

Tani, J., Takaga, T., and Qiu, J., 1998. Intelligent material systems: application of functional materials. Applied Mechanics Reviews 51(8): 505-521.

Tiersten, H.F., 1969. Linear piezoelectric plate vibrations, New York, Plenum Press.

Wetherhold, R.C. and Aldraihem, O.J., 2001. Bending and twisting vibration control of flexible structures using piezoelectric materials. Shock and Vibration Digest 33(3): 187-197.

Yang, J.S. and Batra, R.C., 1994. Free vibration of a piezoelectric body. Journal of Elasticity 28(3): 373-388. 